\title{
On Hyperbolic Jacobsthal-Lucas Sequence
}

\author{
Sait Taş
}

Department of Mathematics, Faculty of Science, Atatürk University, Erzurum, Turkey

\section{Article Info}

Keywords: Hyperbolic numbers, Hyperbolic Jacobsthal-Lucas numbers, Jacobsthal numbers

2010 AMS: 11B37, 11B39, 11B83,

$11 B 99$

Received: 28 June 2021

Accepted: 23 December 2021

Available online: 11 February 2021

\begin{abstract}
In this study, we define the hyperbolic Jacobsthal-Lucas numbers and we obtain recurrence relations, Binet's formula, generating function and the summation formulas for these numbers.
\end{abstract}

\section{Introduction and preliminaries}

In this study, we introduce hyperbolic Jacobsthal-Lucas numbers and give some properties of them. Firstly, we present some background information about hyperbolic numbers and Jacobsthal-Lucas numbers. One can see [1]-[8] for details. Jacobsthal-Lucas sequence $J_{n}$ is defined by the second-order recurence relation

$$
J_{n+2}=J_{n+1}+2 J_{n}
$$

with initial values $J_{0}=2, J_{1}=1$. The first few terms of this sequence are given as follows:

$$
2,1,5,7,17,31,65,127,257,511,1025,2047, \ldots
$$

Binet's formula and generating function of Jacobsthal-Lucas sequence are given by

$$
J_{n}=2^{n}+(-1)^{n}
$$

and

$$
\sum_{n=0}^{\infty} J_{n} x^{n}=\frac{2-x^{2}}{1-x-2 x^{2}}
$$

respectively.

The set of hyperbolic numbers $H$ can be described as

$$
H=\left\{z=x+h y: h \notin R, h^{2}=1, x, y \in R\right\} .
$$

Addition, substruction and multiplication of any two hyperbolic numbers $z_{1}$ and $z_{2}$ are defined by

$$
\begin{aligned}
& z_{1} \pm z_{2}=\left(x_{1}+h y_{1}\right) \pm\left(x_{2}+h y_{2}\right)=\left(x_{1} \pm x_{2}\right)+h\left(y_{1} \pm y_{2}\right) \\
& z_{1} \times z_{2}=\left(x_{1}+h y_{1}\right) \times\left(x_{2}+h y_{2}\right)=x_{1} x_{2}+y_{1} y_{2}+h\left(x_{1} y_{2}+y_{1} x_{2}\right)
\end{aligned}
$$


and the division of two hyperbolic numbers are given by

$$
\frac{z_{1}}{z_{2}}=\frac{x_{1}+h y_{1}}{x_{2}+h y_{2}}=\frac{\left(x_{1}+h y_{1}\right)\left(x_{2}-h y_{2}\right)}{\left(x_{2}+h y_{2}\right)\left(x_{2}-h y_{2}\right)}=\frac{x_{1} x_{2}+y_{1} y_{2}}{x_{2}^{2}-y_{2}^{2}}+h \frac{x_{1} y_{2}+y_{1} x_{2}}{x_{2}^{2}-y_{2}^{2}} .
$$

The hyperbolic conjugation of $z=x+h y$ is defined by

$$
\bar{z}=x-h y .
$$

For more information and properties related hyperbolic numbers, see [9]-[18].

\section{Hyperbolic Jacobsthal-Lucas sequence}

In [14], author presented hyperbolic Fibonacci sequence and examined its properties. In this study, we define hyperbolic Jacobsthal-Lucas sequence and examined some of its properties.

The hyperbolic Jacobsthal-Lucas numbers are defined by

$$
H J_{n}=J_{n}+h J_{n+1}
$$

with initial conditions $H J_{0}=2+h, H J_{1}=1+5 h$ where $h^{2}=1$. Then the first few terms of hyperbolic Jacobsthal-Lucas numbers are

$$
2+h, 1+5 h, 5+7 h, 7+17 h, 17+31 h, 31+65 h, 65+127 h, \ldots,
$$

It can be easily shown that

$$
H J_{n}=H J_{n-1}+2 H J_{n-2} .
$$

In fact, by using the definition of the hyperbolic Jacobsthal-Lucas numbers, we have

$$
\begin{aligned}
H J_{n} & =J_{n}+h J_{n+1}=J_{n-1}+2 J_{n-2}+h\left(J_{n}+2 J_{n-1}\right) \\
& =2 J_{n-2}+h 2 J_{n-1}+J_{n-1}+h J_{n} \\
& =H J_{n-1}+2 H J_{n-2} .
\end{aligned}
$$

Theorem 2.1. Let $\mathrm{HJ}_{n}$ be $n$-th hyperbolic Jacobsthal-Lucas number, then we obtain

$$
\lim _{x \rightarrow \infty} \frac{H J_{n+1}}{H J_{n}}=2
$$

Proof. We have

$$
\lim _{x \rightarrow \infty} \frac{J_{n+1}}{J_{n}}=2 .
$$

for the Jacobsthal-Lucas sequence $J_{n}$. Then using this value for the hyperbolic Jacobsthal-Lucas $H J_{n}$, we get

$$
\begin{aligned}
\lim _{x \rightarrow \infty} \frac{H J_{n+1}}{H J_{n}} & =\lim _{x \rightarrow \infty} \frac{J_{n+1}+h J_{n+2}}{J_{n}+h J_{n+1}} \\
& =\lim _{x \rightarrow \infty} \frac{J_{n+1}+h\left(J_{n+1}+2 H J_{n}\right)}{J_{n}+h J_{n+1}} \\
& =\lim _{x \rightarrow \infty} \frac{\left(\frac{J_{n+1}}{J_{n}}\right)+h\left(\left(\frac{J_{n+1}}{J_{n}}\right)+2\right)}{1+\left(h \frac{J_{n+1}}{J_{n}}\right)} \\
& =\frac{2+4 h}{1+2 h}=2 .
\end{aligned}
$$

Theorem 2.2. The Binet formula for the hyperbolic Jacobsthal-Lucas numbers is given by

$$
H J_{n}=(1+2 h) 2^{n}+(1-h)(-1)^{n} .
$$

Proof. By using the Binet formula of the Jacobsthal-Lucas numbers

$$
J_{n}=2^{n}+(-1)^{n},
$$

we get

$$
\begin{aligned}
H J_{n} & =J_{n}+h J_{n+1} \\
& =2^{n}+(-1)^{n}+h\left(2^{n+1}+(-1)^{n+1}\right) \\
& =(1+2 h) 2^{n}+(1-h)(-1)^{n} .
\end{aligned}
$$


Theorem 2.3. The generating function for the hyperbolic Jacobsthal-Lucas sequence is given by

$$
\sum_{n=0}^{\infty} H J_{n} x^{n}=\frac{2+h+(1-4 h) x}{1-x-2 x^{2}} .
$$

Proof. Let

$$
g(x)=\sum_{n=0}^{\infty} H J_{n} x^{n}
$$

be generating function of hyperbolic Jacobsthal-Lucas numbers. Then we have the following equations:

$$
\begin{array}{r}
g(x)=H J_{0}+H J_{1} x+H J_{2} x^{2}+H J_{3} x^{3}+H J_{4} x^{4}+\ldots \\
-x g(x)=-H J_{0} x-H J_{1} x^{2}-H J_{2} x^{3}-H J_{3} x^{4}-H J_{4} x^{5}-\ldots \\
-2 x^{2} g(x)=-2 H J_{0} x^{2}-2 H J_{1} x^{3}-2 H J_{2} x^{4}-2 H J_{3} x^{5}-2 H J_{4} x^{6}-\ldots \\
\left(1-x-2 x^{2}\right) g(x)=H J_{0}+\left(H J_{1}-H J_{0}\right) x .
\end{array}
$$

By rewriting the last equation, we get

$$
g(x)=\frac{2+4 h+(1-4 h) x}{1-x-2 x^{2}}
$$

with $H J_{0}=2+h, H J_{1}=1+5 h$.

Theorem 2.4. (Catalan's identity) The following identitiy holds for all natural numbers $n$ and $m$ :

$$
H J_{n+m} H J_{n-m}-H J_{n}^{2}=(-1+h)\left[(-2)^{n+m}+(-2)^{n-m}+(-2)^{n+1}\right] .
$$

Proof. By using the formula (2.1), we obtain

$$
\begin{gathered}
H J_{n+m} H J_{n-m}-H J_{n}^{2}=\left((1+2 h) 2^{n+m}+(1-h)(-1)^{n+m}\right)\left((1+2 h) 2^{n-m}+(1-h)(-1)^{n-m}\right) \\
-\left((1+2 h) 2^{n}+(1-h)(-1)^{n}\right)^{2} \\
=\left((5+4 h) 2^{2 n}+(2-2 h)(-1)^{2 n}+(-1+h) 2^{n}(-1)^{n}\left[2^{m}(-1)^{-m}+2^{-m}(-1)^{m}\right]\right) \\
\quad-\left((5+4 h) 2^{2 n}+(2-2 h)(-1)^{2 n}+2(-1+h) 2^{n}(-1)^{n}\right) \\
=(-1+h)\left[(-2)^{n+m}+(-2)^{n-m}+(-2)^{n+1}\right] .
\end{gathered}
$$

Theorem 2.5. (d'Ocagne's identity) The following identitiy holds for any integers $n$ and m:

$$
H J_{m+1} H J_{n}-H J_{m} H J_{n+1}=3(-1+h)\left[(-2)^{m}(-1)^{n}-(-2)^{n}(-1)^{m}\right] .
$$

Proof. By the Binet formula (2.1), we get

$$
\begin{aligned}
H J_{m+1} H J_{n}-H J_{m} H J_{n+1}= & \left((1+2 h) 2^{m+1}+(1-h)(-1)^{m+1}\right)\left((1+2 h) 2^{n}+(1-h)(-1)^{n}\right) \\
& -\left((1+2 h) 2^{m}+(1-h)(-1)^{m}\right)\left((1+2 h) 2^{n+1}+(1-h)(-1)^{n+1}\right) \\
= & 3(-1+h)\left[(-2)^{m}(-1)^{n}-(-2)^{n}(-1)^{m}\right] .
\end{aligned}
$$

Theorem 2.6. (Gelin-Cesaro's identity) The following identitiy holds for any integers $n$ and $m$ :

$$
H J_{n+2} H J_{n+1} H J_{n-1} H J_{n-2}-H J_{n}^{4}=\frac{9}{8}(-1+h)(-2)^{n}\left[(2)^{2 n+1}-13(1-h)(-2)^{n}+4(1-h)\right] .
$$

Proof. Using

$$
\begin{gathered}
H J_{n}=(1+2 h) 2^{n}+(1-h)(-1)^{n}, \\
H J_{n}=(1+2 h)\left[2^{n}+(-1+h)(-1)^{n}\right],
\end{gathered}
$$

and by setting $a=2^{n}, b=(-1+h)(-1)^{n}$ we obtain following values:

$$
\begin{aligned}
& 1 . H J_{n+2}=(1+2 h)[4 a+b] \\
& 2 . H J_{n+1}=(1+2 h)[2 a-b] \\
& 3 \cdot H J_{n-1}=(1+2 h)\left[\frac{a}{2}-b\right]
\end{aligned}
$$


4.HJ $J_{n-2}=(1+2 h)\left[\frac{a}{4}+b\right]$

from the above values, we can easily calculate

$$
\begin{aligned}
H J_{n+2} H J_{n+1} H J_{n--1} H J_{n-2}-H J_{n}^{4} & =(1+2 h)^{4}\left[\left(8 a^{2}-2 a b-b^{2}\right)\left(\frac{a^{2}}{8}+\frac{a b}{4}-b^{2}\right)-\left(a^{4}+b^{4}+4 a^{3} b+6 a^{2} b^{2}+4 a b^{3}\right)\right] \\
& =\frac{9}{8}(-1+h)(-2)^{n}\left[(2)^{2 n+1}-13(1-h)(-2)^{n}+4(1-h)\right] .
\end{aligned}
$$

Theorem 2.7. (Melham's identity) The following identity holds for any integers $n$ and $m$ :

$$
H J_{n+1} H J_{n+2} H J_{n+6}-H J_{n+3}^{3}=9(1-h)(-2)^{n}\left[2^{n+3}+10(1-h)(-1)^{n}\right] .
$$

Proof. Using

$$
\begin{gathered}
H J_{n}=(1+2 h) 2^{n}+(1-h)(-1)^{n}, \\
H J_{n}=(1+2 h)\left[2^{n}+(-1+h)(-1)^{n}\right],
\end{gathered}
$$

and by setting $a=2^{n}, b=(-1+h)(-1)^{n}$ we obtain following values:

1.HJ $J_{n+1}=(1+2 h)[2 a-b]$,

2. $H J_{n+2}=(1+2 h)[4 a+b]$,

3. $H J_{n+6}=(1+2 h)[64 a+b]$,

4. $H J_{n+3}=(1+2 h)[8 a-b]$.

From the above values, we can easily calculate

$$
\begin{aligned}
H J_{n+1} H J_{n+2} H J_{n+6}-H J_{n+3}^{3} & =(1+2 h)^{3}\left[\left(8 a^{2}-2 a b-b^{2}\right)(64 a+b)-(8 a-b)^{3}\right] \\
& =(1+2 h)^{3} 9 a b[8 a-10 b] \\
& =9(1-h)(-2)^{n}\left[2^{n+3}+10(1-h)(-1)^{n}\right] .
\end{aligned}
$$

Theorem 2.8. For $n \geq 0$, we obtain

$$
\sum_{k=0}^{n} H J_{k}=\frac{1}{2}\left(H J_{n+2}-(1+5 h)\right) .
$$

Proof. We use the mathematical induction on $n$. For $n=0$, we have

$$
H J_{0}=\frac{1}{2}\left[H J_{2}-(1+5 h)\right]=\frac{1}{2}[5+7 h-1-5 h]=2+h .
$$

Now assume that it is true for $n=k$, namelyand by setting

$$
\sum_{k=0}^{k} H J_{k}=\frac{1}{2}\left(H J_{k+2}-(1+5 h)\right) .
$$

From the induction hypothesis, we obtain

$$
\begin{aligned}
\sum_{k=0}^{k+1} H J_{k} & =\frac{1}{2}\left(H J_{k+2}-(1+5 h)\right)+H J_{k+1} \\
& =\frac{1}{2}\left(H J_{k+2}-(1+5 h)+2 H J_{k+1}\right) \\
& =\frac{1}{2}\left(H J_{k+3}-(1+5 h)\right) .
\end{aligned}
$$




\section{Conclusion}

The hyperbolic Jacobsthal-Lucas numbers with initial conditions $H J_{0}=2+h, H J_{1}=+5 h$ are defined by

$$
H J_{n}=J_{n}+h J_{n+1}
$$

where $h^{2}=1$.

In this paper, we give the hyperbolic Jacobsthal Lucas numbers and present some recurrence relations, Binet's formula, generating function and some special idetities for these numbers.

\section{Acknowledgements}

The authors would like to express their sincere thanks to the editor and the anonymous reviewers for their helpful comments and suggestions.

\section{Funding}

There is no funding for this work.

\section{Availability of data and materials}

Not applicable.

\section{Competing interests}

The authors declare that they have no competing interests.

\section{Author's contributions}

All authors contributed equally to the writing of this paper. All authors read and approved the final manuscript.

\section{References}

[1] A. F. Horadam, Jacobsthal represantation numbers, Fibonacci Quart., 34 (1996), 40-54.

[2] A. F. Horadam, Jacobsthal and Pell curves, Fibonacci Quart., 26 (1988), 79-83.

[3] A. F. Horadam, Jacobsthal representation polynomials, Fibonacci Quart., 35 (1997), 137-148.

[4] A. F. Horadam, Basic properties of a certain generalized sequence of numbers, Fibonacci Quart., 3(3) (1965), 161-176.

[5] K. Atanassov, Remark on Jacobsthal numbers, Part 2. Notes Number Theory Discrete Math., 17(2) (2011), 37-39.

[6] K. Atanassov, Short remarks on Jacobsthal numbers, Notes Number Theory Discrete Math., 18(2) (2012), 63-64.

[7] M. C. Dikmen, Hyperbolic Jacobsthal numbers, Asian Res. J. Math., 4 (2019), 1-9.

[8] S. Tas, The Hyperbolic Quadrapell sequences, Eastern Anatolian J. Sci. VII(I) (2021), 25-29.

[9] M. A. Güngör, A. Cihan, On dual-hyperbolic numbers with generalized Fibonacci and Lucas numbers components, Fundamental J. Math. App., 2(2) (2019), 162-172

[10] A. P. Stakhov, Gazale formulas, a new class of the hyperbolic Fibonacci and Lucas functions, and the improved method of the 'Golden' Cryptograph, Academy of Trinitarism, 77(6567) (2006), 1-32.

[11] A. P. Stakhov, I. S. Rozin, Hyperbolic Fibonacci trigonometry, Rep. Ukr. Acad. Sci., 208 (1993), 9-14, [In Russian].

[12] A. P. Stakhov, B. Tkachenko, On a new class of hyperbolic functions, Chaos Solitons Fractals, 23 (2005), 379-389.

[13] F. Falcon, A. Plaza, The k-Fibonacci hyperbolic functions, Chaos Solitons Fractals, 38(2) (2008), 409-420.

[14] F. T. Aydın, Hyperbolic Fibonacci sequence, Univers. J. Math. Appl., 2(2) (2019), 59-64.

[15] S. Halic1, On bicomplex Jacobsthal-Lucas numbers, J. Math. Sci. Model., 3(3) (2020), 139-143.

[16] H. Gargoubi, S. Kossentini, f-algebra structure on hyperbolic numbers, Adv. Appl. Clifford Algebras, 26(4) (2016), 1211-1233.

[17] A. E. Motter, A. F. Rosa, Hyperbolic calculus, Adv. App. Clifford Algebras, 8(1) (1998), 109-128.

[18] L. Barreira, L. H. Popescu, C. Valls, Hyperbolic sequences of linear operators and evolution maps, Milan J. Math., 84 (2016), $203-216$. 